\title{
Individual-based modelling of resource competition to predict density-dependent population dynamics: a case study with white storks
}

\author{
Damaris Zurell, Ute Eggers, Michael Kaatz, Shay Rotics, Nir Sapir, Martin Wikelski, Ran Nathan \\ and Florian Jeltsch
}

D. Zurell (damaris.zurell@wsl.ch), U. Eggers and F. Jeltsch, Inst. of Biochemistry and Biology, Univ. of Potsdam, Maulbeerallee 2, DE-14469 Potsdam, Germany. DZ also at: Landscape Dynamics, Swiss Federal Research Inst. WSL, Zürcherstrasse 111, CH-8903 Birmensdorf, Switzerland. FJ also at: Berlin-Brandenburg Inst. of Advanced Biodiversity Research (BBIB), DE-14195 Berlin, Germany-M. Kaatz, Vogelschutzwarte Storchenhof Loburg e.V., Chausseestr. 18, DE-39279 Loburg, Germany. - S. Rotics and R. Nathan. Dept of Ecology, Evolution and Behavior, The Hebrew Univ. of Jerusalem, Edmond J. Safra Campus, IL-91904 Jerusalem, Israel. - N. Sapir and M. Wikelski, Dept of Migration and Immuno-Ecology, Max Planck Inst. for Ornithology, Schlossallee 2, DE-78315 Radolfzell, Germany. MW also at: Dept of Biology, Konstanz Univ., DE-78315 Konstanz, Germany.

\begin{abstract}
Density regulation influences population dynamics through its effects on demographic rates and consequently constitutes a key mechanism explaining the response of organisms to environmental changes. Yet, it is difficult to establish the exact form of density dependence from empirical data. Here, we developed an individual-based model to explore how resource limitation and behavioural processes determine the spatial structure of white stork Ciconia ciconia populations and regulate reproductive rates. We found that the form of density dependence differed considerably between landscapes with the same overall resource availability and between home range selection strategies, highlighting the importance of fine-scale resource distribution in interaction with behaviour. In accordance with theories of density dependence, breeding output generally decreased with density but this effect was highly variable and strongly affected by optimal foraging strategy, resource detection probability and colonial behaviour. Moreover, our results uncovered an overlooked consequence of density dependence by showing that high early nestling mortality in storks, assumed to be the outcome of harsh weather, may actually result from density dependent effects on food provision. Our findings emphasize that accounting for interactive effects of individual behaviour and local environmental factors is crucial for understanding density-dependent processes within spatially structured populations. Enhanced understanding of the ways animal populations are regulated in general, and how habitat conditions and behaviour may dictate spatial population structure and demographic rates is critically needed for predicting the dynamics of populations, communities and ecosystems under changing environmental conditions.
\end{abstract}

Our natural world is rapidly changing, substantially impacting individual organisms, populations, communities and ecosystems in complex ways. Predicting species response to environmental change has consequently become a prominent issue in modern ecology and modelling has become a key tool for making quantitative predictions regarding the fate of populations and communities under different environmental scenarios (Nathan et al. 2011, Zurell et al. 2012, Jeltsch et al. 2013). Understanding population regulation is crucial for predicting its dynamics (Sinclair 2003, Benton 2012) and consequently its functioning within communities and ecosystems. Density regulation is a negative feedback mechanism that leads to decreases in key demographic rates like survival, recruitment and reproduction as density increases. Due to the ongoing rapid global climate changes and increased habitat fragmentation, there is currently a pressing need to improve understanding of density effects and incorporate these into predictions of population dynamics (Best et al. 2007).

Though populations may be limited by top-down processes such as predation or by factors such as disturbances, the most basic mechanisms behind population regulation in animals are bottom-up and relate to resource availability and competitive behavioural mechanisms (Newton 1998, Sinclair 2003, Benton 2012), for example through depletion of resources or through interference (from simple disturbance of prey to fighting and food-robbing). In heterogeneous landscapes, the distribution of resources and other limiting factors determine habitat quality and thereby may influence animal fitness (survival and/or reproductive success). Density-regulating mechanisms acting within populations limit the maximum population size that can be sustained in a given environment, usually termed the carrying capacity. Both resource distribution and competitive behavioural 
mechanisms may thus determine the structure of animal populations and their spatial distribution. These factors are key to understanding density-dependence processes, regardless of animal size, surrounding environment and medium (sea, land, air), diet, territoriality, spatial distribution and locomotion capabilities.

Traditional models of population and community dynamics are often based on 'mean field' assumptions where demographic parameters are scaled by overall density, and encounters between individuals occur in proportion to abundance (Morales et al. 2010). While this assumption may be valid in homogenous environments or at very coarse scale, mean field predictions are unlikely to accurately capture local dynamics in heterogeneous environments or to predict the consequences of future environmental conditions for which no data or contemporary analogues exist. A different approach is taken by process-based models using 'first principles' that portray how life history traits, ecophysiological and behavioural mechanisms and constraints interact with the animal's changing environment. Here, focus is naturally on the individual level since individual organisms constitute the key element of ecological systems, and system-level properties such as population dynamics and demographic rates arise from behaviour of individuals and interactions between them (Grimm and Railsback 2005, Evans et al. 2012). Individual-based models (IBMs) allow not only the simulation of individual behaviour but also modelling different constraints on their abilities (e.g. their sensory and motion capacities). Moreover, IBMs simulate decision-making processes in which individuals aim to optimize their fitness (e.g. survival or number of offspring) by adjusting their behaviour to match their internal state and the environment within which they live (Nathan et al. 2008).

So far, not many studies have explicitly modelled how behavioural mechanisms and spatial resource use link to population dynamics in a bottom-up way (Morales et al. 2010). In recent years, several studies used spatially explicit, individual-based models to simulate home ranges resulting from individual movement and behaviour, and from the interactions between individuals (Mitchell and Powell 2004, 2012, Moorcroft et al. 2006, Buchmann et al. 2011, Nabe-Nielsen et al. 2013). These models aim to make spatially explicit predictions of individual's space-use by modelling animal movement, for example correlated random walks (Moorcroft et al. 2006), and resource consumption (Mitchell and Powell 2004, 2012, Buchmann et al. 2011, 2012, Nabe-Nielsen et al. 2013). A few models explicitly linked movement and space-use to population dynamics. Using an individual-based model of the Iberian lynx Lynx pardinus, Revilla and Wiegand (2008) showed that movement behaviour and survival interact dynamically and have profound effects on local population and metapopulation dynamics. On a local scale, Johst et al. (2001) developed an individualbased model to study patch choice of foraging white storks Ciconia ciconia and the impact of different land use patterns on food supply and post-hatching breeding success. Wang and Grimm (2007) studied density dependence of home range dynamics in common shrews Sorex araneus by systematically varying the initial population densities. They showed that dynamic space-use in animals can have strong effects on population regulation and should thus be included in realistic population models.

To derive and predict the exact form of densitydependence in demographic rates one needs to know the carrying capacity. Mitchell and Powell (2004) hypothesised that if a minimum resource threshold must be met to allow the establishment of a home range, then resource depression by single individuals ultimately sets a limit to the number of viable home ranges a landscape can support. Thus, carrying capacity is reached if the landscape becomes unable to support any additional home ranges that meet the minimum resource requirement. Mitchell and Powell (2012) followed this incentive to estimate the carrying capacity for black bears. Hayes et al. (2007) and Buchmann et al. $(2011,2012)$ provide examples for drift-feeding salmonids and for entire animal communities, respectively. Hitherto, no study stressed beyond predicting spatial structuring of populations (or communities) and carrying capacity and studied how density dependence in demographic rates emerges from spatial resource use of individuals in heterogeneous landscapes. Although many studies model density dependence emergent from competition over resources, most of these focus on temporal dynamics in resource availability (Benton 2012, Johst et al. 2013) rather than on the effects of spatial resource heterogeneity on density dependent population dynamics.

Our objectives in this study were, thus, to predict a) the spatial population structure in heterogeneous landscapes as a function of resource availability and ecophysiological and behavioural constraints, b) the maximum carrying capacity of landscapes, and c) emergent density-dependent population dynamics using an individualbased model. Mitchell and Powell (2004) hypothesised that animals try to optimise resource acquisition within their home range through selection of resource patches, analogous to optimal foraging theory (Stephens and Krebs 1986). Fretwell and Lucas (1970) proposed the concept of ideal free distributions which assumed that habitats in a landscape vary in quality or suitability and that suitability declines with increasing population density. Thus, individuals sequentially fill available habitat starting with the best patches (Newton 1998). Following these concepts, we designed the model so that the landscape is sequentially filled by individuals that choose the optimal home range given the available resources constrained by their ecophysiological (e.g. resource requirements) and behavioural properties (e.g. home range selection strategy) until carrying capacity is reached. As the landscape is filled up by individual home ranges, resource distribution changes through resource depression. After estimating the carrying capacity of a landscape, the density level can be controlled in the model by setting the initial number of individuals to a pre-defined proportion below carrying capacity. Once the individuals have successfully established a home range and found a mate, they may reproduce and fulfil their day-to-day energy demands through foraging. The model predicts density-dependence in reproductive success. Rearing young induces seasonal changes in the resource requirements within the home ranges, potentially leading to deaths of young and adult individuals within the population (Mitchell and Powell 2004). 
We constructed and parameterised the model for breeding white storks Ciconia ciconia because there is a relative wealth of data from this species reported in previous studies (Johst et al. 2001). The white stork is a charismatic species of general conservation concern in Europe that has often been used to illustrate aspects of population ecology based on rich long-term datasets of high quality (Tryjanowski et al. 2009). Johst et al. (2001) already developed a spatially explicit, individual based foraging model for white storks on which we built our study. However, Johst et al. (2001) only simulated a single white stork breeding pair and did not take into account the effects of intraspecific competition. We thus extend their physiology- and behaviour-based model to simulate entire populations with individuals interacting through competition for resources in a spatially explicit landscape.

In our model, home range selection and foraging patch selection are nested. Individuals aim to fulfil their daily energy demands by optimising the selection of foraging patches within the home range. Home ranges are optimised by choosing the central home range cell best suited to support daily, central-place foraging. Here, we test two alternative, nested, strategies for optimal foraging and optimal home range selection and their effects on spatial population structure, carrying capacity and density-dependent reproductive success. Animals forage among patches within their home range according either to a time-minimising strategy where individuals seek to maximise their net energy intake per time, or according to an energy-maximising strategy where they simply seek to maximise energy intake (Stephens and Krebs 1986). For both strategies, individuals need to acquire enough resources to fulfil their daily energy requirements, and are limited by energy and time costs for flight and foraging. Individuals that forage according to a time-minimising strategy, also use an equivalent strategy for home range optimisation, an area-minimising strategy, meaning that they choose that cell as central home range cell that allows the highest net energy intake per time (the highest energy rate). Similarly, individuals foraging according to an energy-maximising strategy will choose as central nest site that cell which allows highest net energy gain in absolute energy units, which is called the resourcemaximising home range strategy (cf. Mitchell and Powell 2004). For either strategy, a central nest site can only be established if individuals are able to fulfil their daily energy demands by foraging trips that means if the home range satisfies a resource threshold which is the sum of metabolic, foraging and flight costs. We hypothesise that home ranges in heterogeneous landscapes should be larger when individuals use the resource-maximising home range strategy because they do not trade off energy gain against time costs but will fly to more distant cells with higher time and flight costs if those cells provide higher net resource levels. Larger home ranges should lead to lower carrying capacities.

Coloniality has been observed in storks (Vergara and Aguirre 2006) and other birds, and we thus test the effect of colonisation behaviour on population dynamics and density dependence. Actively forming local nest aggregations may lead to deviations from an ideal free distribution because individuals will not necessarily fill the habitat sequentially but will prefer a nest site with sufficient resources within an existing colony over the most optimal nest site. This may prevent optimal exploitation of resources which should lead to lower carrying capacity. The spatial distribution of home ranges should be strongly affected by the spatial distribution of resources and, thus, the degree of fragmentation in the landscape. We hypothesise that higher resource clumping will lead to more overlap in home ranges while nest sites will be more evenly distributed in highly fragmented landscapes. However, because individuals sequentially fill habitats according to or close to an ideal free distribution, the form of density dependence in reproductive rates should not be influenced by spatial population structure and, thus, by landscape fragmentation.

We will run all comparisons in theoretical, fractal landscapes. However, to evaluate whether the tested home range strategies can reproduce observed relationships between breeding success and density, we will compare model predictions of reproductive success and corresponding breeding pair numbers across different density levels against longterm monitoring data of white stork breeding populations in Eastern Germany (Eggers et al. unpubl.).

\section{Methods}

\section{Study organism}

The white stork is a large, soaring avian migrant with conspicuous roosts and nest sites, mostly in human environments. In central Europe, white storks arrive from the wintering grounds around March to April and start breeding shortly thereafter. White storks are semi-altricial birds and nestlings stay in the nest and are fed by their parents until 60-70 days of age (Jovani and Tella 2004). A female can lay up to 6 eggs but on average clutch sizes are approximately 3-4 eggs (Kosicki 2010). Both parents share breeding and parental care. White storks are opportunistic foragers and generalists and forage on both vertebrates and invertebrates. They forage diurnally and solitary but, in their central European breeding range, may be seen in small groups of 10 individuals or more if prey is abundant. Fights for food resources are uncommon.

\section{Basic model description}

The model description follows the ODD (overview, design concepts and details) protocol for describing individualbased models (Grimm et al. 2006, 2010). The entire model was implemented in $\mathrm{C}++$ and its code is available in the expanded online edition.

\section{Purpose}

The purpose of the model is to predict the spatial structure and breeding success of white stork populations in heterogeneous landscapes by explicitly simulating foraging behaviour and home range formation of competing individuals. Because resource depletion is modelled explicitly, the model can predict the maximum carrying capacity of white stork breeding populations in different landscapes, and densitydependent breeding success by inducing fixed stork density levels below carrying capacity. 


\section{Entities, state variables and scale}

The biological entities of the model are individual white storks that are characterised by the following state variables: energy levels (required energy per day, consumed energy), pairing status (partner or no partner), identity of the partner, reproductive state (breeding or non-breeding), the number of dependent nestlings, the age-related energy levels of their nestlings (required and consumed energy), and the coordinates of the nest site. The spatial entities of the model are square cells of a landscape grid that are characterised by the maximum daily resource level and the actual, depleted resource level. The model landscape is a square grid of variable size ( $33 \times 33$ cells in the standard simulations) with $0.5 \mathrm{~km}$ resolution. Edge effects are avoided by assuming cyclic boundary conditions, so that individuals leaving the grid at one edge reappear on the opposite edge. That way, we mimic continuous space use where individuals may also forage outside the study area and where individuals from outside may also exploit resources within the study area. The temporal resolution is one day; hence energy demands of white storks and energy acquisition are compared on a daily basis. Likewise, resource levels of each cell are renewed on a daily basis, under the simplified assumption that overall prey abundance and accessibility are constant throughout the season. Resource depletion (reduction of a cell's actual resource level during one day) can be interpreted as reduced detection probability of the prey due to repeated scaring by the foragers. Foraging is simulated in shorter time steps (minutes). After home range establishment, simulations are run for 60 days corresponding to the time period that nestlings are fed in the nest (Jovani and Tella 2004).

\section{Process overview and scheduling}

The core part of our model is a foraging module simulating single foraging trips of individual white storks that was introduced by Johst et al. (2001). Generally, individuals make several foraging trips per day to satisfy their daily energy requirements and those of the nestlings. White storks are typical central-place foragers and are only active during daytime, using $18 \mathrm{~h} \mathrm{day}^{-1}$ for foraging in central European summer. In breeding storks caring for a brood, a single foraging trip may last up to $2 \mathrm{~h}$ after which they return to the nest for feeding (Johst et al. 2001). There are time costs for the flight depending on the distance between the nest and the foraging patch. The energy intake is determined by the quality of the patch and the time used for foraging discounted for the travel costs. During foraging, the actual resource level of the cell is reduced by the amount of energy taken by the storks foraging in each cell. Thus, after each foraging trip of an individual the actual resource landscape will look different within one simulation day. Storks can repeatedly forage in a cell during one day until all the resources are depleted.

Entire model runs are characterised by two distinct phases: 1) home range selection and incubation, and 2) a post-hatching, nestling rearing phase (Fig. 1).

For each stork entering the simulation, all grid cells are tested as potential central nest sites. For each of the potential nest sites, a typical foraging day of the focal stork is simulated during which it makes several foraging trips to fulfil its

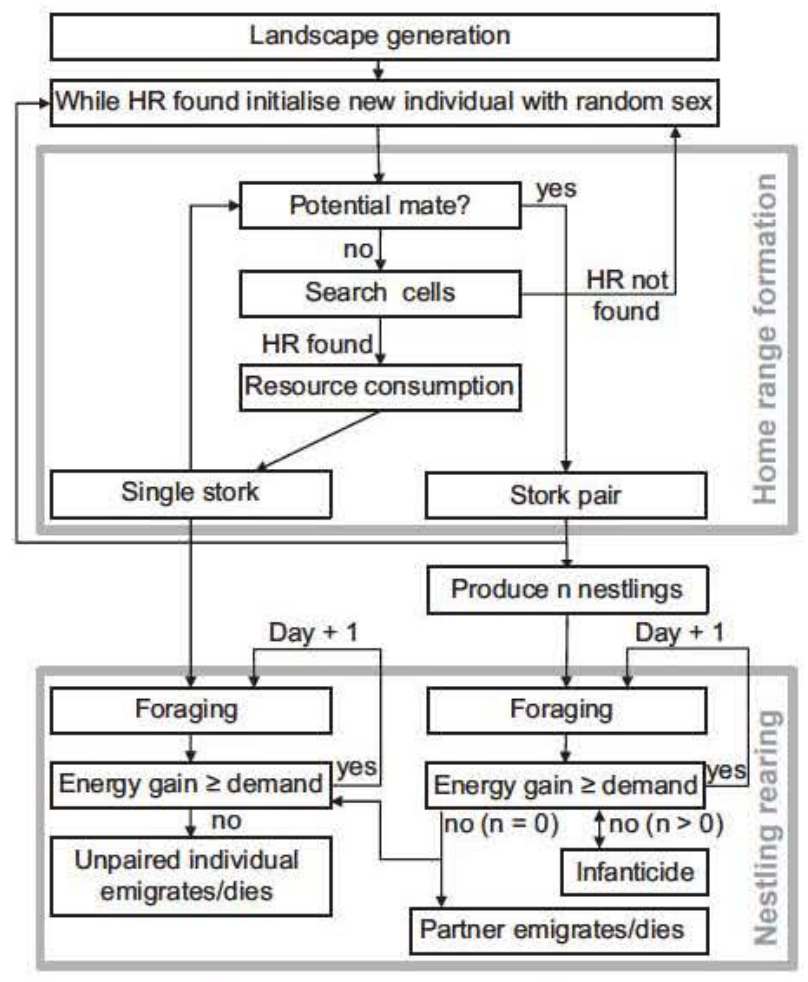

Figure 1. Flowchart of individual-based stork model. (HR: home range).

energy demands. These include metabolic energy demands and pre-hatching extra energy costs for nest building, egg producing and laying, and incubation (Djerdali et al. 2008). The stork is then assigned to the optimal nest site given available resources, whereby optimisation criteria depend on the chosen home range selection strategy. We thus assume that potential nest sites (e.g. rooftops, chimneys and electricity pylons) are available anywhere in the landscape. Then, foraging from the chosen nest site is repeated and the resources in the foraging patches are depleted according to stork food intake. Thus, the resource landscape perceived by any new stork entered into the simulation will look different. The sex of new storks is chosen randomly, and new storks will first look for a potential (unpaired) partner already occupying a nest site. A breeding pair is formed if a partner of the opposite sex is found, and only breeding pairs lay eggs in the pre-hatching phase and raise nestlings in the post-hatching phase.

During the post-hatching phase, the model is run for 60 days during which the nestlings are fed by the parents. Each day the adults undertake several foraging trips to provide food for the young and for themselves (for resting metabolism, flight and foraging). Thereby, the order in which storks forage is chosen randomly for each day. At the end of each day, the sum of the energy intake of the adults is compared to the energy requirements of the adults and the (growing) nestlings. If the energy demands exceed the energy supply, the number of nestlings is reduced to simulate starvation and/or infanticide (Tortosa and Redondo 1992). If pairs without nestlings, including pairs that have lost their nestlings, cannot fulfil their daily energy demands, they will split up and eventually disperse (Fig. 1), although both adults and 
nestlings have a tolerance rate against starvation (Supplementary material Appendix 1 Table A1). Due to insufficient self-thermoregulation, nestlings are brooded during the first 20 days after hatching (Jovani and Tella 2004), meaning that one adult always remains at the nest and thus the available foraging time per adult per day is halved.

\section{Design concepts}

\section{Emergence}

The spatial distribution of nest sites (home ranges) and the pairs' reproductive success emerge from the model. The location of the nest sites is driven by home range optimisation. Reproductive success is determined by the amount of available food which depends on population density and individual foraging behaviour. Maximum travelling distances to optimal foraging patches and, thus, home range sizes emerge from the model and depend on time and flight costs and available resources. Initial clutch sizes, energy requirements of the nestlings and the adults as well as movement capacity and costs are represented by empirical rules and parameters (Supplementary material Appendix 1 Table A1).

\section{Adaptive behaviour and objectives}

Individuals show adaptive behaviour (decision making) when foraging and when selecting the optimal central home range cell. Their objectives are defined by their home range selection strategy and their foraging strategy which are nested. Either storks forage according to a time-minimising principle and select home ranges according to an area-minimising strategy, or they forage according to an energy-maximising principle and select home range according to a resource-maximising strategy. Because it is not known how closely storks forage to an optimal solution, we assume a proportion of random patch selection of $25 \%$ which can be interpreted as the maximum error rate in decision making (cf. Johst et al. 2001). Random patch selection is defined by empirical parameters and means that suitable foraging patches within the home range are chosen randomly with the constraint that storks preferentially select nearby patches (defined as $2.5 \mathrm{~km}$ from the nest site; Johst et al. 2001) with an empirical probability of $72 \%$ and that randomly chosen patches are no farther than $5 \mathrm{~km}$ from the nest (Supplementary material Appendix 1 Table A1).

\section{Prediction and sensing}

Within home range selection, the individuals explicitly calculate the potential energy gain and associated movement costs for each potential nest site. During foraging, individuals explicitly calculate the expected energy gain discounted for movement and time costs for each cell within the home range. To do so, individuals sense the amount of food available in the grid cells.

\section{Interaction}

Individuals interact indirectly through the depletion of resources on a daily basis. We focus here only on intraspecific competition for food resources, assuming, for simplicity, that intraspecific competition for other resources and inter-specific interactions have negligible effects on the availability of food resources, mortality rates and breeding success of white storks.

\section{Stochasticity}

Stochasticity is included in our model by several means. The sex ratio and the proportion of random patch selection are probabilistic and compared against a random number drawn from a uniform distribution. With random patch selection, available foraging patches are chosen randomly whereby the proportion of nearby versus distant patches is again probabilistic.

\section{Observation}

From the model, we observed all nest locations, the total number of individuals and pairs in each cell, and the number of nestlings for each nest. In principal, the model also allows observing the entire movement paths of each individual but these aspects were beyond the scope of our study.

\section{Initialisation}

The simulations start with home range formation and are initialized with one stork. Then, more storks with randomly chosen sex are added until 1) carrying capacity $K$ is reached or until 2) a pre-defined number of individuals $N$ inhabit the landscape. In 1$), K$ is reached when resources are depleted to an extent that new individuals entered into the simulation are not able to find a home range with enough resources to support their daily energy requirements. In 2), various levels of intraspecific competition are simulated by first letting the model run until carrying capacity to determine $K$ and then running simulations with fixed maximum numbers of individuals according to $N / K$ ratios of 0.9 to 0.1 (in 0.1 decrements), respectively. After home range formation is completed, the post-hatching, nestling rearing, phase is initialized with four nestlings per breeding pair (Kosicki 2010).

\section{Input}

The amount of resources in the landscape is the only environmental condition currently considered in the model. This requires a landscape grid characterized by an index of resource productivity. Weather conditions are currently not considered as the literature provides no quantitative or direct physiological information regarding how incubation and nestling or adult survival are affected by weather (Jovani and Tella 2004). For the resource landscape, neutral landscapes were generated using the well-established midpoint displacement algorithm (Saupe 1988). This fractal algorithm creates a three-dimensional surface, a topographical map, characterized by two parameters, the Hurst-factor $H$ controlling the degree of spatial autocorrelation (habitat clumping) and the variance $\sigma^{2}$ in the displacement of points (With 1997). A proportion $p$ of the lowest $z$-values was set to zero (unsuitable patches); $z$-values of the remaining, suitable patches were rescaled to range between zero and one in order to describe the relative resource productivity of the landscape, with one being the most productive landscape. Average resource productivity across the suitable patches was set to 40 units for all landscapes to ensure comparability. Thus, each landscape allowed a maximum energy intake rate of $53200 \mathrm{~kJ} \mathrm{~h}^{-1}$ (per $33 \times 33$ cells). Default process and landscape parameters are given in Supplementary material Appendix 1 Table A1. Foraging parameters were taken from Johst et al. (2001) and 
updated by GPS data of nine free ranging storks obtained during the 2011 breeding season. Detailed descriptions of the processes, submodels, and data are provided in Supplementary material Appendix 1.

\section{Simulations and sensitivity analysis}

We ran a number of simulations to test the effects of behavioural strategies and landscape fragmentation, and a local sensitivity analysis to assess the robustness of the model predictions against changes in parameter values. Because the model includes stochasticity, for each model configuration we ran 100 simulations to obtain the distribution of model outputs from which the variation due to stochasticity was estimated.

Foremost, an area-minimising strategy of home range selection and a resource-maximising strategy were compared. In addition, we tested the effect of actively forming local nest aggregations (Vergara and Aguirre 2006). Under this scenario, new storks entered into the simulation started home range search in already colonised cells and only extended their search to un-colonised cells if resources in colonised areas were depleted below their demands. This was compared to scenarios without attraction to local nest aggregations where storks were free to choose the best available nest site from all cells. Furthermore, we compared model predictions for landscapes with different degrees of fragmentation. To this end, we simulated fractal landscapes with varying Hurst factors $H$ that controls spatial autocorrelation with higher $H$ corresponding to greater habitat clumping. We tested $H=0.1 / 0.3 / 0.5 / 0.9$ (default landscape with $H=0.7$, Supplementary material Appendix 1 Table A1). To check for stochasticity in landscape structure we created 10 replicate fractal landscapes for each fragmentation degree generated with the same fractal parameters.

In the local sensitivity analysis, key parameters (Supplementary material Appendix 1 Table A1) were changed by $\pm 50 \%$ of their default values while keeping all other parameters constant. The scenario of storks following an area-minimising home range strategy and actively forming local nest aggregations served as baseline for the sensitivity analysis. In the case of extra pre-hatching energy requirements, the default parameter was changed by $\pm 100 \%$ to check the impact of not accounting for energy costs of nest building, egg-laying and incubation. Also, nestling starvation tolerance rates were changed by $\pm 100 \%$ to check the impact of instant nestling starvation on model results. The proportion of random patch selection (defaults to $25 \%$ ) was also changed by $\pm 100 \%$ to check the differences in spatial population structure and mean reproductive success for extreme behaviours. Because the sex of simulated individuals is randomly allocated, Allee effects, resulting from unequal proportions of females and males in the population (Allee 1931, Stephens et al. 1999), are possible by chance. To control for Allee effects, we also ran scenarios with equal numbers of females and males entering the simulation in turn. Additional scenarios were run without brooding of the early nestlings to assess the consequences of raising young at weather conditions that do not incur thermoregulatory costs and without risk of predation.

\section{Results}

\section{Carrying capacity and spatial population structure}

The predicted mean breeding densities for the different home range selection strategies in default and replicate landscapes range from 5 to 10 breeding pairs per $100 \mathrm{~km}^{2}$ and correspond to mean densities reported for central Europe in moderately farmed to low populated areas (Kosicki 2010; for Tables of simulation results see Supplementary material Appendix 2 Tables A2-A5). Thereby, mean breeding densities for populations following an area-minimising home range strategy were generally higher than those for the resource-maximising strategy. The predicted spatial population structure was highly variable between different behavioural scenarios (cf. Supplementary material Appendix 2 Table A4). As could be expected, pronounced differences in spatial structuring were found between populations forming local nest aggregations and populations without local aggregating. Local nest aggregation led to much higher local breeding pair numbers while the breeding pairs were more evenly spread across the landscape and more cells were occupied with nests in scenarios without local nest aggregations (Fig. 2a-b, Supplementary material Appendix 2 Fig. A1a-b, Table A4). Also, the local mean fledgling numbers were higher in scenarios without local nest aggregations (Fig. 2c-d, Supplementary material Appendix 2 Fig. A1c-d). Nevertheless, mean breeding pair numbers and mean fledgling numbers for the entire landscape were only slightly higher for scenarios without local aggregating with maximum differences of 0.21 mean breeding pairs per $100 \mathrm{~km}^{2}$ (Supplementary material Appendix 2 Table A2). Generally, more central nest sites in terms of position within the resource landscape produced more fledglings in area-minimising home ranges (Fig. 2c) while this could not be found for resourcemaximising home ranges (Fig. 2d). For the latter strategy, much more cells were occupied with nest sites (Supplementary material Appendix 2 Table A4). Although fledgling numbers per pair in resource-maximised home ranges were higher than for the area-minimising strategy (1.46 compared to 1.36; Supplementary material Appendix 2 Table A2), absolute fledgling numbers were lower due to higher nest failures (25 fledglings compared to 36; Supplementary material Appendix 2 Table A2). High fragmentation led to more evenly dispersed nest sites within the resource landscapes and, consequently, more cells were occupied by nest sites though this effect was much weaker than differences due to different home range strategies (Supplementary material Appendix 2 Tables A4-A5).

\section{Reproductive success and nestling mortality}

On average, at carrying capacity the breeding pairs produced 1.36-1.47 fledglings from an initial number of four nestlings meaning that, due to resource limitations, only $34-37 \%$ of the hatched nestlings fledged. Empirical (Jovani and Tella 2004) and theoretical (Johst et al. 2001) studies suggested that the highest nestling mortality occurs within the first 20 days of life. In our simulations, nestling mortality within the first 20 days of life averaged $85-93 \%$ of all nestling deaths during the entire 60 days nestling-rearing phase for the area-minimising home range strategy and $91-97 \%$ for 

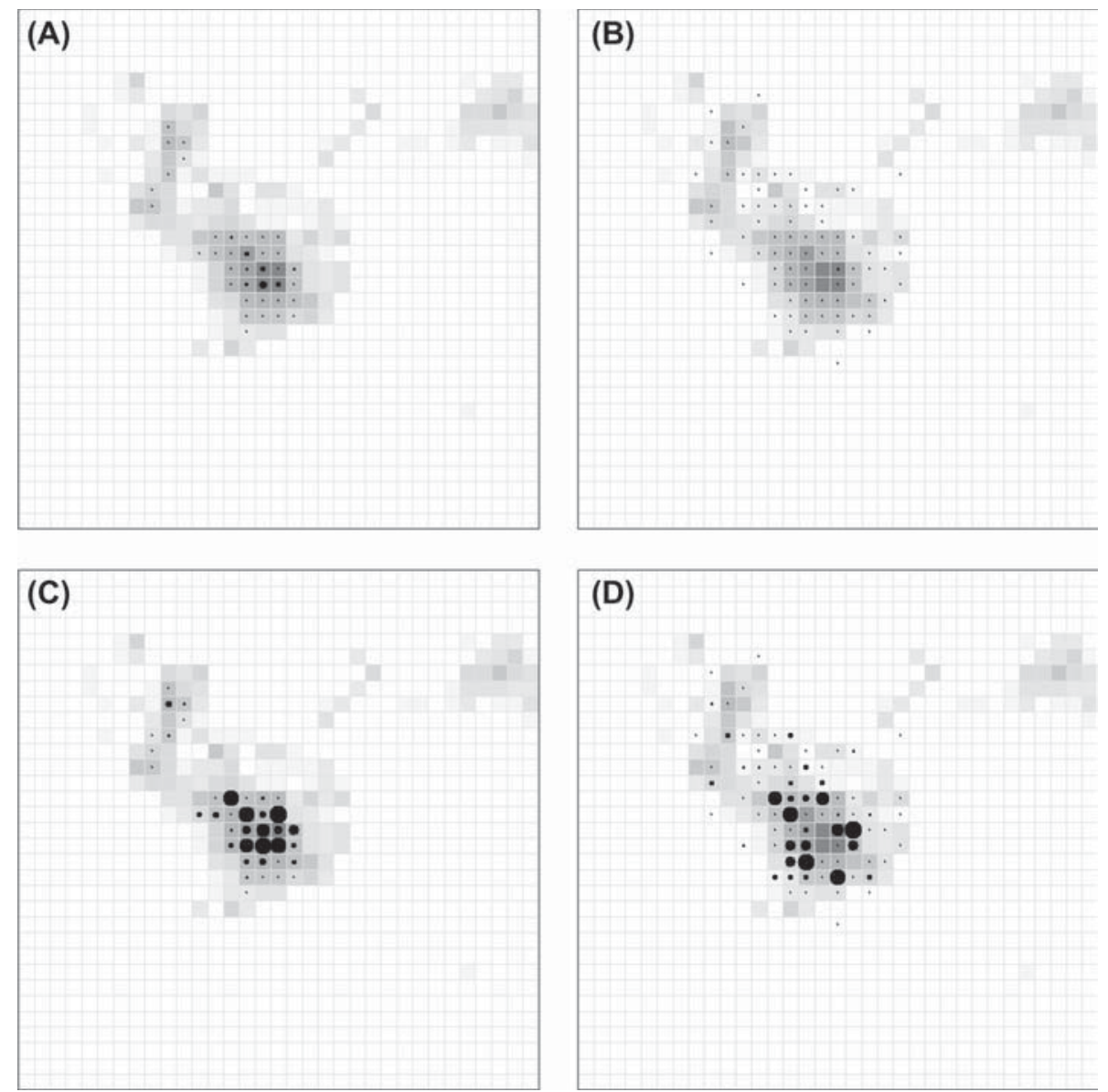

Figure 2. Spatial population structure of white stork breeding populations in the default landscape. Left panels depict a stork population following an area-minimising home range strategy $(\mathrm{A}, \mathrm{C})$, right panels a stork population following a resource-maximising home range strategy (B, D). Storks do not actively form local nest aggregations. Grey shading indicate resource levels $\left[\mathrm{kJ} \mathrm{h}^{-1}\right]$. Points in the upper panels show mean numbers of breeding pairs for $\mathrm{n}=100$ simulations and points in the lower panels show mean number of fledglings (nestlings having reached maturity) per breeding pair. Point size is proportional to the relative numbers, scaled for each row separately, and corresponds to a maximum of (A) 3.3 and (B) 1.13 mean breeding pairs, and to a maximum of (C) 1.64 and (D) 1.66 mean fledglings per breeding pair.

the resource-maximising home range strategy (Supplementary material Appendix 2 Table A2). In comparison, Jovani and Tella (2004) reported $91 \%$ of deaths within the first 20 days of life. Generally, early nestling mortality was lower for scenarios with lower brooding intensity (as adults had more time for foraging and feeding). Yet, the brooding strategy did not change the overall post-hatching breeding success at the end of the breeding season, but merely affected the onset of highest nestling mortality (Fig. 3). However, prolonged brooding beyond the first 20 days of nestlings' life markedly reduced the expected number of fledglings. Predicted mean breeding densities, mean fledgling numbers and nestling survival rates at carrying capacity were very robust across replicate landscapes (Fig. 4, Supplementary material Appendix 2 Table A2).

\section{Density dependence in reproductive success}

The mean number of fledglings increased under decreasing population density, although this effect was highly variable for different home range selection strategies and across replicate landscapes, and not always as pronounced. For the resource-maximising home range strategy, maximum reproductive success at low densities, and thus the steepness of the density dependence curve, was generally lower than for the area-minimising home range strategy (Fig. 4a-b). At 10\% density, the number of fledglings for storks following an areaminimising strategy ranged 2.3-3.7 in replicate landscapes, and ranged only 1.4-2.5 fledglings for storks following a resource-maximising home range strategy. Overall, for the default parameter settings where we assumed an error rate in detection of optimal foraging patches of $25 \%$ (expressed as proportion of random patch selection), fledgling rates in replicate landscapes varied considerably at lower densities while being quite constant at high densities (Fig. 4a-b). We found this pronounced effect of landscape on density regulation across all degrees of fragmentation tested (Supplementary material Appendix 2 Fig. A2). Generally, mean nestling survival rates at low densities were higher when storks did not actively form local nest aggregations. However, a far greater effect was exerted by the proportion of random patch selection. When assuming perfect detection of optimal foraging patches, then much higher nestling survival rates could be observed for both home range strategies, especially at low 
(A)

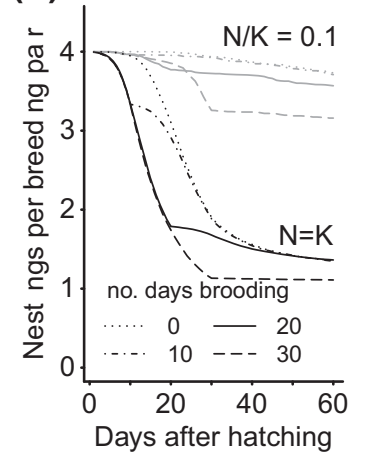

(B)

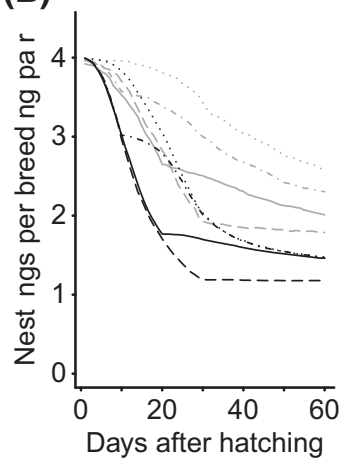

Figure 3. Development of mean number of nestlings per breeding pair during the nestling-rearing phase (from hatching to fledging) in the default landscape for different densities and for different brooding durations. (A) depicts stork populations following an area-minimising home range strategy with local aggregations, (B) populations following a resource-maximising home range strategy with local aggregations.
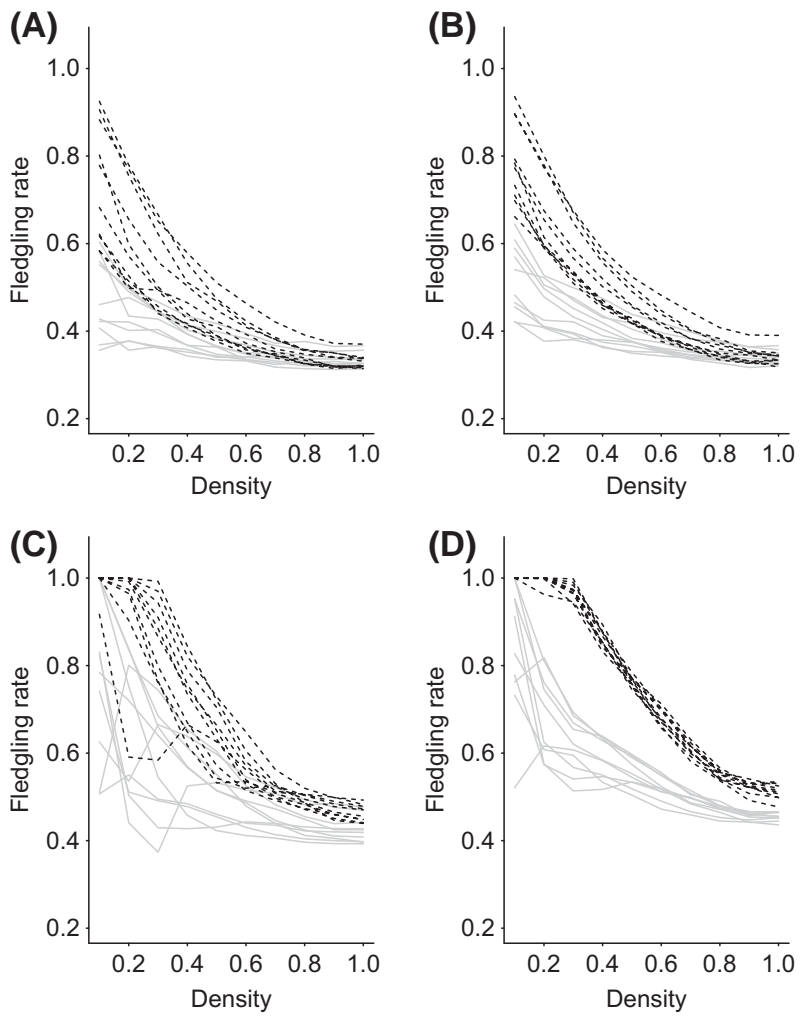

Figure 4. Fledgling rates (mean proportion of nestlings surviving the breeding season) plotted as a function of density, for different home range selection strategies and for 10 replicate landscapes (with default fractal parameters). Dashed lines indicate areaminimising home range selection, grey solid lines resourcemaximising home range selection. Left panels (A, C) depict population forming local aggregations, right panels $(\mathrm{B}, \mathrm{D})$ populations without local aggregation. Top panels (A, B) show simulations run with default parameter settings with a proportion of random patch selection (error rate in detection of optimal foraging patch) of $25 \%$. Lower panels (C, D) assume perfect detection of optimal foraging patches. For each replicate landscape we $\operatorname{ran} n=100$ replicate simulations. densities (Fig. 4c-d). In simulations without local nest aggregation and with perfect detection ability, the form of density dependence in replicate landscapes converged to an inverse sigmoidal curve for the area-minimising home range strategy while it was still highly variable for resource-maximising (Fig. 4d). As can be expected, landscapes that produced low fledgling numbers at low density levels exhibited higher proportions of nest failures (\% unsuccessful pairs).

Figure 5 compares predicted fledgling numbers and fledging rates against the number of breeding pairs in replicate landscapes across all simulated density levels and corresponding long-term monitoring data from the state of Brandenburg in Germany (Eggers et al. unpubl.). Both tested home range strategies show a linear relationship between fledgling numbers and breeding pairs as was found empirically (Fig. 5a-c), but under-prediction of fledgling numbers increases towards higher breeding pair numbers. For the area-minimising home range strategy, perfect detection of optimal foraging patches led to over-prediction of fledgling numbers for lower breeding pair numbers. We observed a clear negative relationship between fledging rates and breeding pair numbers in the simulation results (Fig. 5b, d). This trend was accompanied by a high degree of scatter due to variations in carrying capacity and form of density dependence across landscapes. The monitoring data exhibit a similar pattern for high fledgling rates but also contain a positive trend between low fledgling rates and the number of breeding pairs. This trend is not apparent in the mean simulation results (though rudimentary for extreme values, not shown). Quantitatively, the monitoring data showed a broader range of fledgling rates and a higher mean absolute value of 2.61 fledglings per breeding pair than most simulations. Only scenarios assuming area-minimised home ranges with perfect detection of optimal foraging patches were able to adequately reproduce fledgling rates for high breeding pair numbers but showed less scatter in fledgling rates than empirical data and other simulation scenarios.

\section{Sensitivity analysis}

Sensitivity analysis indicated that in our model breeding density at carrying capacity was most sensitive to energetic constraints. Breeding density strongly increased with maximum energy intake rates and, correspondingly, decreased with increasing metabolic energy requirements. Both decreasing and increasing pre-hatching (nest building, egg producing and laying, and incubation) energy costs negatively affected breeding density; the former through increased nest failure rates (\% non-breeding pairs), and the latter because higher resource requirements led to lower colonisation rates. In general, population trends under different density levels were robust across wide parameter space. The sensitivity of fledging success and early nestling mortality was the highest for the nestling tolerance rate against starvation and for the pre-hatching energy requirements. However, although high nestling tolerance rates against starvation led to higher fledging rates, the absolute number of fledglings decreased considerably due to higher nest failures. Variation in key model outputs through changing grid sizes were within the range of variation across replicate landscapes. Mean demographic rates were highly robust against degree of landscape fragmentation. 


\section{Discussion}

The predictive model developed here allows exploring how spatial resource availability and individual behaviour interact to shape the spatial structuring of white stork populations and density-dependent population dynamics. We found that the form of density dependence in demographic rates differed strongly between landscapes and was strongly affected by home range selection strategies, colonisation behaviour and by errors in optimal foraging. As expected from theories of density-dependence, populations at carrying capacity exhibited similar reproductive success rates regardless of the absolute number of breeding pairs or their exact spatial structure. Spatial population structure was largely determined by behavioural mechanisms like the tendency to form local nest aggregations and the home range selection strategy. Overall, our model results highlight the interactive effects of landscape and behaviour on the spatial structuring of populations and in particular on the spatial structuring of demographic rates. For understanding species-specific population dynamics, it is often more interesting how demography evolves, for example across the reproductive season. Here, our results suggest that high nestling mortality in storks soon after hatching, which is often assumed to be the outcome of harsh weather, may also result from density regulatory effects on food provision. In the following, we will discuss general implications for density dependence in demographic rates and population dynamics, and some specific implications for white stork population dynamics.

\section{Carrying capacity and density dependence}

Landscape carrying capacity was an emergent property of the model resulting from physiological and behavioural constraints. The exact spatial population structure was strongly affected by behavioural mechanisms, foremost by the home range selection strategy but also by colonisation behaviour, i.e. the tendency to actively form local nest aggregations. As hypothesised, we found significantly lower carrying capacities for breeding pairs for the resource-maximising compared to the area-minimising home range strategy. This was mainly result of higher nest failure rates throughout the season rather than result of lower settling rates per se. Also, carrying capacity for populations actively forming local nest aggregations was lower than for populations freely choosing the best available nest sites, though only slightly. We found support for our hypothesis that breeding pairs should be more evenly spread across the landscape when fragmentation is high because home range overlap will decrease. As overall resource availability was the same for all fragmentation levels, the range of carrying capacities of landscapes across different degrees of fragmentation did not significantly differ.

In accordance with theories of density dependence, overall nestling survival rates at carrying capacity were very robust across replicate landscapes and between home range strategies. If the birds forage in or close to an ideal free distribution manner, they should distribute themselves between different habitats in proportion to the amount of resources available such that the fitness of individuals in each habitat is equal (Fretwell and Lucas 1970, Parker 1978). Here, we did not look at life-time fitness but only on reproductive success in one year as a short-term fitness proxy. We found, nevertheless, that irrespective of the absolute number of breeding pairs that a landscape can sustain, for populations at carrying capacity, we can expect similar maximum fledgling rates across landscapes.

Our results further support the theoretical prediction of a progressive increase in mean breeding performance as density and thus, interference decreases. Yet, our results also indicate that this effect is not always pronounced and that the form of density dependence is highly landscape specific and also strongly affected by behavioural properties (Fig. 3-4). In some cases, local resources were insufficient or the exploitation of local resources was inefficient to support the energy demands of the growing nestlings, resulting in comparably low fledgling numbers and increased nest failure rates even at low densities. This picture is consistent across different landscape fragmentation scenarios and may be observed for both home range selection strategies tested here. Nevertheless, maximum reproductive success at low densities is generally lower for the recourse-maximising home range strategy than for area-minimisation because resource gain is not traded off against costs for flight and foraging. Setting an upper limit to the distance moved by storks following a resource-maximising strategy, may decrease differences in the forms of density dependence.

The form of density dependence seems very sensitive to errors made in detection of optimal foraging patches and to colonisation behaviour. Detection probability alters the form of density dependence in demographic rates but not equally for different home range selection strategies. While perfect detection led to convergence of density-dependent nestling survival rates across landscapes for the areaminimising home range strategy, the form of densitydependent survival rates was still highly variable between landscapes for the resource-maximising strategy. Although perfect knowledge of the resource landscapes is often a key assumption of optimality models, it can reasonably be assumed that animals never forage optimally because of incomplete knowledge of the resource distributions in new or variable environments. When individuals enter an unknown environment they first have to learn the key environmental parameters. However, because the environment may be highly variable, for example in the course of one season, or because of unexpected weather events or human forcing, animals may not be able to learn enough of their environment to allow optimal foraging (McNamara and Houston 1985). Our results imply that home range selection strategy, detection probabilities in optimal foraging patches and fine-scale resource distribution in the landscape interactively affect the form of density dependence. Density-dependent demographic rates seem to be more sensitive to fine-scale resource variability for certain home range selection or optimal foraging strategies. To define general rules, more home range selection and foraging behaviours need to be tested (e.g. cost minimisation and activity minimisation; Sih 1984) over a continuum from heterogeneous to homogeneous landscapes.

Colony-building has been observed in many bird species and was also reported for storks (Vergara and Aguirre 2006). Often, however, it is not clear whether this is an active behaviour or an emergent property. Our simulations indicate that 
local colonies may also arise if individuals are free to choose their nest sites depending solely on resource availability although this effect strongly depends on the home range selection strategy and was not apparent for resource-maximised home ranges. Apparently, active colonisation behaviour may lead to deviations from an ideal free distribution of nest sites in heterogeneous landscapes with strong effects on the form of density dependence in demographic rates. At high population density, demography is rather insensitive to colonisation behaviour. At lower population density, however, the form of density dependence is highly landscape-specific if an ideal free distribution is not entirely met. Thus, demography is tightly linked not only to density and overall resource availability but is very sensitive to behavioural strategies, the degree to which resource exploitation is optimal, and to actual, fine-scale spatial resource distribution. Hence, assessing and predicting demographic rates and population dynamics requires taking into account interacting effects of individual behaviour and the environment (Johst et al. 2001). In particular, the efficiency of individual foraging and the behavioural strategies for optimal foraging and home range selection may have substantial consequences for overall population dynamics.

\section{White stork population dynamics}

Our modelling results also provide specific and new insights on white stork breeding ecology. It is known that white storks suffer from high early nestling mortality (Jovani and Tella 2004, Denac 2006). This has been commonly advocated to insufficient thermoregulatory capacity of nestlings below the age of 20 days making them vulnerable to stressful conditions like cold and rainy weather (Jovani and Tella 2004). Yet, also resource-related causes have been discussed. For example, Denac (2006) suggested that weather effects may be mediated by food resource abundance. In their simulation study, Johst et al. (2001) showed that nestling mortality may also be related to food supply and, in particular, that the age at which nestlings die due to starvation is determined by the time span of increasing energy requirements of the nestlings in relation to food supply. They predicted that in low quality landscapes most nestlings will die before the age of 20 days and that this age of high nestling mortality should increase with increasing quality of the landscape. Our analyses point to density regulation in post-hatching breeding success as another complicating mechanism. While Johst et al. (2001) only modelled a single breeding pair, we simulated here populations at different densities. Early nestling mortality rates were well within empirically observed ranges reported in the literature (Jovani and Tella 2004). Overall, our results suggest that high early nestling mortality may also solely result from density regulatory effects and not only from weather effects as often assumed. We do not claim that weather may not substantially affect white stork reproductive success, but rather emphasise that density dependent effects may play a more prominent role in shaping early nestling mortality than often assumed. In reality, weather and density dependence probably interact to determine vital rates (Sxther et al. 2005, Boyce et al. 2006), and these processes may be of variable importance in different years. Furthermore, in contrast to Johst et al. (2001), our model predicts that in populations at carrying capacity, high early nestling mortality can be expected irrespective of the quality of the landscape.

We did not explicitly include any weather effects in our model, but ran idealised scenarios with different levels of brooding. Thereby, at high densities brooding intensity only shifted the time of the highest nestling mortality while mean fledging success at the end of the breeding season did not differ between the different scenarios if the brooding period did not exceed 20 days after hatching. Prolonged brooding beyond this time span would result in reduced fledging success for this season. Thus, there seems to be a strong tradeoff between the duration of parental care and reproductive output.

We found that carrying capacities may vary between similar landscapes leading to some degree of scatter in reproductive success against breeding pair numbers which we also found in long-term monitoring data (Fig. 5). However, the empirical findings contain not only a negative trend, a density effect as our simulations imply, but also a positive trend between fledging rates and the number of breeding pairs. This positive trend in fledging rates was not captured
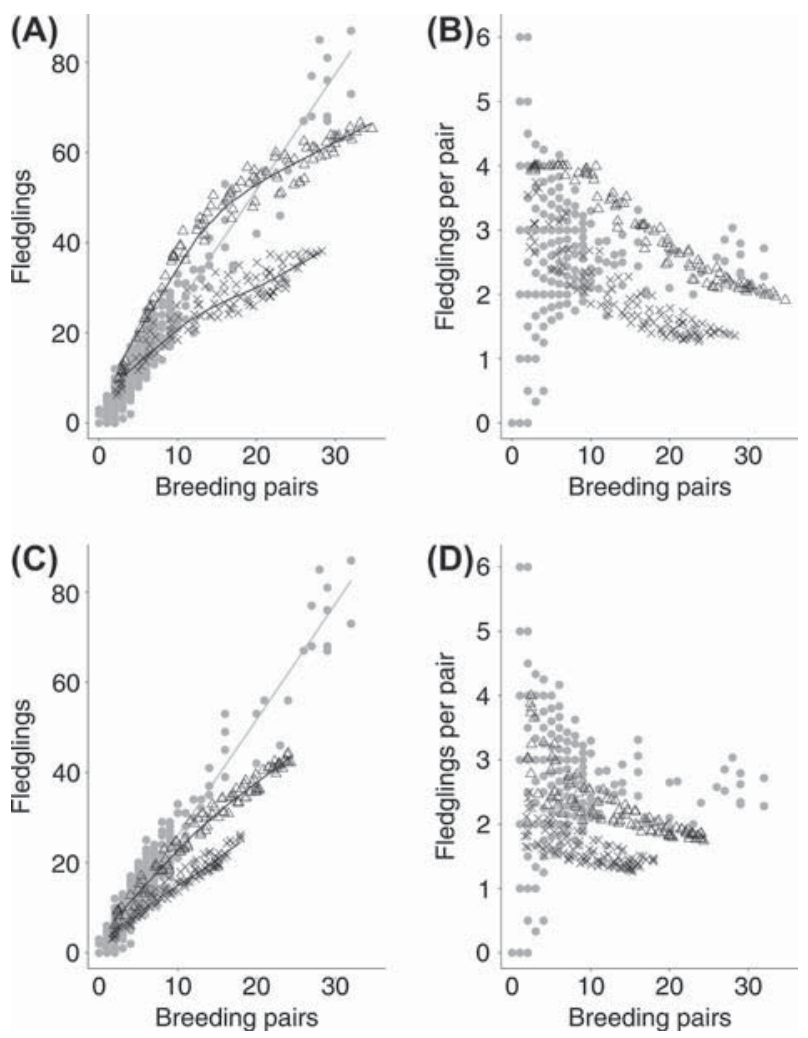

Figure 5. The number of fledglings (A, C) and the number of fledglings per breeding pair (B, D) plotted against number of breeding pairs. Grey dots indicate long-term monitoring data of white stork breeding performance in Brandenburg (State)/Germany from 1956-2009 (Eggers et al. unpubl.; see Supplementary material Appendix 1 for detailed data description). Black symbols indicate simulation results. Top panels (A-B) show storks following an areaminimising home range strategy without local nest aggregations, lower panels show storks following a resource-maximising home range strategy without local nest aggregations. Plus signs indicate default parameterisation with a proportion of random patch selection of $25 \%$, triangles indicate perfect detection of optimal foraging patches. Shown are the mean numbers within $n=100$ simulations across replicate landscapes (with default fragmentation) and across density levels. 
by our model because it does not yet incorporate any direct mortality sources that may act on the nestlings within real populations. Such direct mortality sources are not only the aforementioned rainy weather effects, but also accidental falling of the nests, stochastic adult mortality and nest predation, although the latter two are probably negligible in white storks. Moreover, the mean fledging rates observed in Brandenburg were higher than those simulated by our model except for the area-minimising home range strategy with perfect detection of optimal foraging patches. On the one hand, density-dependent mortality rates may be mediated by dynamic changes in resource availability and distribution not yet included in our model. For example, an upswing in resource availability or quality during times of increased energy requirements of the nestlings may mitigate nestling mortality rates (resource-tracking hypothesis; Forbes and Mock 2000). On the other hand, the sensitivity analysis pointed to nestling tolerance against starvation as a highly sensitive parameter affecting fledging rates and early nestling mortality. Empirical evidence suggests that nestlings may survive longer periods of starvation of up to one week (Tortosa and Redondo 1992, Kaatz unpubl.) which may also explain the higher overall fledging rates found in Brandenburg. Nevertheless, we emphasise that although our model is simplified to represent only foraging and no other environmental effects, and parameters such as starvation tolerance are not calibrated, it still reproduced basic patterns that are similar to those reported from empirical studies (Fig. 3, 5).

\section{Outlook}

Overall, our model provides the grounds for mechanistically and dynamically estimating carrying capacities of landscapes as well as current density status of populations and for predicting density-dependent reproductive rates. Because population dynamics and demographic rates are not imposed by empirical parameters but emerge from individual behaviour and intraspecific competition for food resources, the model is not restricted to specific populations but allows prediction over a wide range of environmental conditions. Although our model was specifically built for breeding white storks, its design is highly generic and can easily be adapted for different situations and other animal species by adapting the foraging module including the movement rules. The model incorporates simple biological mechanisms related to individual resource use that are broadly applicable across lifecycle stages and life history attributes. For instance, while here we explored density-dependent reproductive success, the model could also be used to study density regulatory effects at over-wintering sites or across the entire annual cycle of migratory species like the white stork. Due to its modular structure, the ecophysiological and decision-making processes can easily be adjusted and refined to include, for example, physiological responses to weather, or different behavioural responses like competitive mechanisms or other foraging movement modes than central place foraging (Buchmann et al. 2012). By comparing model results to empirical data, for example through the 'multiscope' view of inverse, pattern-oriented modelling (Grimm and Railsback 2012), species-specific behavioural mechanisms such as the optimal foraging strategy may be better understood. Recent advances in tracking and monitoring technologies provide otherwise hard-to-get information on input variables and may facilitate mechanistic understanding, and also stipulate rigorous use of such process-based models to study population dynamics (Nathan et al. 2008). In this respect, theoretical studies as presented here are useful for aggregating current system knowledge and for elucidating information gaps, and may thus stimulate new analyses of existing data or even new field studies and experiments (Jovani and Grimm 2008, Zurell et al. 2010). Overall, the presented modelling approach is valuable for both applied and theoretical ecology as it helps to advance the understanding and prediction of the species' carrying capacities and demographic rates. Moreover, our approach is useful for studying population limitation in animals more generally, in particular how spatial resource use drives density regulation. More thorough analyses are needed to define general rules how behavioural strategies as well as spatial variability and temporal dynamics in resources interact to determine the form of density dependence in demographic rates, and our modelling approach provides the means to do so.

Acknowledgements - We would like to thank Karin Johst and Mike Mitchell for valuable comments that helped to improve the manuscript. We acknowledge the generous support of DIP grants (DFG) NA 846/1-1 and WI 3576/1-1 to RN, MW and FJ. DZ received funding from the People Programme (Marie Curie Actions) of the European Union's Seventh Framework Programme (FP7/20072013) under REA grant agreement no. 624958. RN also acknowledges support from the Adelina and Massimo DellaPergola Chair of Life Sciences and the Minerva Center for Movement Ecology.

\section{References}

Allee, W. C. 1931. Animal aggregations. A study in general sociology. - University of Chicago Press, Chicago.

Benton, T. G. 2012. Individual variation and population dynamics: lessons from a simple system. - Phil. Trans. R. Soc. B 367: 200-210.

Best, A. S. et al. 2007. Which species will successfully track climate change? The influence of intraspecific competition and density dependent dispersal on range shifting dynamics. - Oikos 116: 1531-1539.

Boyce, M. S. et al. 2006. Demography in an increasingly variable world. - Trends Ecol. Evol. 21: 141-148.

Buchmann, C. M. et al. 2011. An allometric model of home range formation explains the structuring of animal communities exploiting heterogeneous resources. - Oikos 120: 106-118.

Buchmann, C. M. et al. 2012. Movement upscaled - the importance of individual foraging movement for community response to habitat loss. - Ecography 35: 436-445.

Denac, D. 2006. Resource-dependent weather effect in the reproduction of the white stork Ciconia ciconia. - Ardea 94: 233-240.

Djerdali, S. et al. 2008. Food supply and external cues limit the clutch size and hatchability in the white stork Ciconia ciconia. - Acta Ornithol. 43: 145-150.

Evans, M. R. et al. 2012. Predictive ecology: systems approaches. - Phil. Trans. R. Soc. B 367: 163-169.

Forbes, S. and Mock, D. W. 2000. A tale of two strategies: life-history aspects of family strife. - Condor 102: 23-34.

Fretwell, S. D. and Lucas, H. L. J. 1970. On territorial behavior and other factors influencing habitat distribution in birds. - Acta Biotheor. 19: 16-36. 
Grimm, V. and Railsback, S. F. 2005. Individual-based modeling and ecology. - Princeton Univ. Press.

Grimm, V. and Railsback, S. F. 2012. Pattern-oriented modelling: a 'multiscope' for predictive systems ecology. - Phil. Trans. R. Soc. B 367: 298-310.

Grimm, V. et al. 2006. A standard protocol for describing individualbased and agent-based models. - Ecol. Modell. 198: 115-126.

Grimm, V. et al. 2010. The ODD protocol: a review and first update. - Ecol. Modell. 221: 2760-2768.

Hayes, J. W. et al. 2007. Process-based modelling of invertebrate drift transport, net energy intake and reach carrying capacity for drift-feeding salmonids. - Ecol. Modell. 207: 171-188.

Jeltsch, F. et al. 2013. How can we bring together empiricists and modellers in functional biodiversity research? - Basic Appl. Ecol. 14: 93-101.

Johst, K. et al. 2001. Foraging in a patchy and dynamic landscape: human land use and the white stork. - Ecol. Appl. 11: 60-69.

Johst, K. et al. 2013. Scaling up: how do exogenous fluctuations in individual-based resource competition models re-emerge in aggregated stochastic population models? - Popul. Ecol. 55: $173-182$.

Jovani, R. and Tella, J. L. 2004. Age-related environmental sensitivity and weather mediated nestling mortality in white storks Ciconia ciconia. - Ecography 27: 611-618.

Jovani, R. and Grimm, V. 2008. Breeding synchrony in colonial birds: from local stress to global harmony. - Proc. R. Soc. B 275: $1557-1564$

Kosicki, J. Z. 2010. Reproductive success of the white stork Ciconia ciconia population in intensively cultivated farmlands in western Poland. - Ardeola 57: 243-255.

McNamara, J. M. and Houston, A. I. 1985. Optimal foraging and learning. - J. Theor. Biol. 117: 231-249.

Mitchell, M. S. and Powell, R. A. 2004. A mechanistic home range model for optimal use of spatially distributed resources. - Ecol. Modell. 177: 209-232.

Mitchell, M. S. and Powell, R. A. 2012. Foraging optimally for home ranges. - J. Mammal. 93: 917-928.

Moorcroft, P. R. et al. 2006. Mechanistic home range models capture spatial patterns and dynamics of coyote territories in Yellowstone. - Proc. R. Soc. B 273: 1651-1659.

Morales, J. M. et al. 2010. Building the bridge between animal movement and population dynamics. - Phil. Trans. R. Soc. B 365: 2289-2301

Nabe-Nielsen, J. et al. 2013. How a simple adaptive foraging strategy can lead to emergent home ranges and increased food intake. - Oikos 122: 1307-1316.
Nathan, R. et al. 2008. A movement ecology paradigm for unifying organismal movement research. - Proc. Natl Acad. Sci. USA 105: 19052-19059.

Nathan, R. et al. 2011. Spread of North-American wind-dispersed trees in future environments. - Ecol. Lett. 14: 211-219.

Newton, I. 1998. Population limitation in birds. - Academic Press.

Parker, G. A. 1978. Searching for mates. - In: Krebs, J. R. and Davies, N. B. (eds), Behavioral ecology: an evolutionary approach. Blackwell, pp. 214-244.

Revilla, E. and Wiegand, T. 2008. Individual movement behavior, matrix heterogeneity, and the dynamics of spatially structured populations. - Proc. Natl Acad. Sci. USA 105: 19120-19125.

Saupe, D. 1988. Algorithms for random fractals. - In: Petigen, H. O. and Saupe, D. (eds), The science of fractal images. Springer, pp. $71-113$.

Sih, A. 1984. Optimal behavior and density-dependent predation. - Am. Nat. 123: 314-326.

Sinclair, A. R. E. 2003. Mammal population regulation, keystone processes and ecosystem dynamics. - Phil. Trans. R. Soc. B 358: 1729-1740.

Stephens, D. W. and Krebs, J. R. 1986. Foraging theory. - Princeton Univ. Press.

Stephens, P. A. et al. 1999. What is the Allee effect? - Oikos 87: 185-190.

Sæther, B.-E. et al. 2005. Generation time and temporal scaling of bird population dynamics. - Nature 436: 99-102.

Tortosa, F. S. and Redondo, T. 1992. Motives for parental infanticide in white storks Ciconia ciconia. - Ornis Scand. 23: 185-189.

Tryjanowski, P. et al. 2009. Severe flooding causes a crash in production of white stork (Ciconia ciconia) chicks across central and eastern Europe. - Basic Appl. Ecol. 10: 387-392.

Vergara, P. and Aguirre, J. I. 2006. Age and breeding success related to nest position in a white stork Ciconia ciconia colony. - Acta Oecol. 30: 414-418.

Wang, M. and Grimm, V. 2007. Home range dynamics and population regulation: an individual-based model of the common shrew Sorex araneus. - Ecol. Modell. 205: 397-409.

With, K. A. 1997. The application of neutral landscape models in conservation biology. - Conserv. Biol. 11: 1069-1080.

Zurell, D. et al. 2010. The virtual ecologist approach: simulating data and observers. - Oikos 119: 622-635.

Zurell, D. et al. 2012. Uncertainty in predictions of range dynamics: black grouse climbing the Swiss Alps. - Ecography 35: 590-603. 